\title{
Mechanisms and Functions of MiR-200 Family in Hepatocellular Carcinoma
}

This article was published in the following Dove Press journal: OncoTargets and Therapy

\author{
Yinqi Mao' \\ Wei Chen' \\ Han Wu' \\ Chenbin Liu' \\ Jingjun Zhang ${ }^{2}$ \\ Shuying Chen $\mathbb{I D}^{3}$ \\ 'School of Medicine, Shanghai Jiao Tong \\ University, Shanghai, People's Republic of \\ China; ${ }^{2}$ Department of Rehabilitation, \\ Huashan Hospital Affiliated to Fudan \\ University, Shanghai, People's Republic of \\ China; ${ }^{3}$ Department of Laboratory \\ Medicine, Huashan Hospital, Fudan \\ University, Shanghai, People's Republic of \\ China
}

\begin{abstract}
Hepatocellular carcinoma (HCC) is one of the most common clinically malignant tumors of the digestive system. It ranks the sixth most common malignant tumor in the world and ranks fourth among cancer-related death worldwide. At present, early diagnosis and prognosis monitoring of hepatocellular carcinoma mainly use alpha-fetoprotein combined with ultrasonography, which leads to clinical frequently missed diagnosis or even misdiagnosis. Therefore, seeking specific diagnostic and monitoring molecules of hepatocellular carcinoma are still hot topics in contemporary medical practice. MicroRNA is an endogenous non-coding small RNA that regulates the expression of the target molecule and participates in various biological processes in vivo. The miR-200 family, the most common celebrity family of microRNAs, is commonly lower expression in a variety of cancers and is closely associated with tumorigenesis and outcome, especially hepatocellular carcinoma. This review mainly discusses the expression changes, specific molecular mechanisms, biological functions and clinical values of miR-200 family in hepatocellular carcinoma. Moreover, we highlighted utilization of miR-200 family as molecular biomarkers for early diagnosis, prognostic monitoring and appropriate therapy in hepatocellular carcinoma.
\end{abstract}

Keywords: hepatocellular carcinoma, miR-200 family, mechanism, biological function, diagnosis, prognosis

\section{Introduction}

Hepatocellular carcinoma (HCC) is one of the most common malignant tumors, which is the fifth most common cancer in men worldwide and seventh in women. ${ }^{1}$ It seriously endangers human physical and mental health due to its characteristics of the hidden disease, high malignant degree and poor prognosis. At present, the clinical treatment of HCC is mainly surgical resection supplemented by chemotherapy and radiotherapy. Researches show that in patients with HCC with a small tumor volume, the recurrence rates at 2 and 5 years after surgical resection are $50 \%$ and $75 \%{ }^{2}$. Lacking specific biomarkers, early diagnosis of HCC is still difficult although breakthrough progress has been gotten in the pathogenesis of HCC in the last few years.

MicroRNA (miRNA), which is a class of small RNAs discovered in recent years, plays the dual role of carcinogenesis and cancer suppression. A large number of studies have reported that there are many abnormally expressed miRNAs in HCC that are related to early diagnosis and treatment, especially the microRNA-200 (miR-200) family. This review will summarize the function of miR-200 family in $\mathrm{HCC}$ by regulating different signaling pathways, their value for early diagnosis of HCC and the possibility as a potential therapeutic target.
Correspondence: Shuying Chen Department of Laboratory Medicine, Huashan Hospital Affiliated to Fudan University, 12 Wulumuqi Middle Road, Shanghai 200040, People's Republic of China

Tel $+861376|73| 807$

$\mathrm{Fax}+8602152888321$

Email xsjrmt@163.com
OncoTargets and Therapy 2020:13 13479-13490

13479

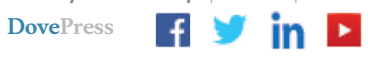

(c) (1) (5) $\odot 2020 \mathrm{Mao}$ et al. This work is published and licensed by Dove Medical Press Limited. The full terms of this license are available at https://www.dovepress.com/terms.php cc. you hereby accept the Terms. Non-commercial uses of the work are permitted without any further permission from Dove Medical Press Limited, provided the work is properly attributed. For permission for commercial use of this work, please see paragraphs 4.2 and 5 of our Terms (https://www.dovepress.com/terms.php). 


\section{Overview of MiRNA}

MiRNAs are a class of conservative non-coding RNAs that are approximately 18 to 22 nucleotides in length. ${ }^{3}$ MiRNAs degrade or inhibit the translation of mRNA by directly binding to the base of the target molecule 3 '-untranslatable region. They regulate more than $60 \%$ of human protein-coding genes as a kind of post-transcriptional regulator. ${ }^{4} \mathrm{~A}$ single miRNA can regulate hundreds of different targets while a single mRNA can also be regulated by multiple different miRNAs. ${ }^{5}$ MiRNAs take part in a series of important processes in life, including early embryonic development, cell proliferation, apoptosis, cell death, fat metabolism, and even regulate the differentiation of stem cells. The diversity of miRNA sequence, structure, abundance and expression pattern makes it possible that miRNA can be used as a regulatory factor of $\mathrm{mRNA}$ encoded proteins, and has an important impact on gene expression, cell cycle regulation and even ontogeny under normal physiological conditions. However, the abnormal expression of miRNAs is closely related to the occurrence and development of human cancer. Mavrakis et al have found miR-19b, miR-20a, miR-26a, miR-92 and miR-223 all promote the occurrence of mice leukemia by establishing NOTCH1 induced T-ALL mouse model through using NOTCH1 and empty vector (control group) or miRNA (experimental group) transduced hematopoietic progenitor cells (HPC) and transplanted HPC into recipients which received lethal irradiation. ${ }^{6}$ Song et al have reported that miR-22 expression enhances the ability of human breast epithelial cells to develop into mammary gland structures indicating that miR-22 triggers epithelialmesenchymal transition (EMT) by transporting transgenic retroviral vector encoding human miR-22 gene into immortalized human breast epithelial cells. ${ }^{7}$ Studies above reveal a causal relationship between miRNA and cancer development. Expression profiling also confirms that different human carcinomas have different miRNA expressions. ${ }^{8}$

\section{Overview of MiR-200 Family}

The MiR-200 family is one of the most common star families in the microRNA system, consisting of 5 highly conservative members (miR-141, miR-200a, miR-200b, miR-200c and miR-429). According to the different positions on the chromosomes, the miR-200 family is divided into two different gene clusters: miR-200a/miR-200b/miR-429 and miR-141/miR200c. MiR-200a, miR-200b and miR-429 are derived from chromosome $1 \mathrm{p} 36.33$ while miR-141 and miR-200c are derived from chromosome $12 \mathrm{p} 13.31 .^{9}$ In addition, they can be also divided into two groups according to their seed sequence, which members of the same group have in common: miR-429/miR-200b/miR-200c and miR-141/miR-200a. The difference between the two groups is the third nucleotide of the seed sequence. The seed sequence of miR-429/miR-200b/ miR-200c group is AAUACUG while the seed sequence of miR-141/miR-200a group is AACACUG (Figure 1). ${ }^{10}$ The differences in genome location and seed sequence make the miR-200 family members undergo differential expression and differential function regulation once they are activated by various exogenous stimuli, which in turn leads to disorders of physiological processes in the body and even the appearance of pathological processes. The earliest study of the correlation between the miR-200 family and human diseases indicates that members of the miR-200 family are necessary for the proper differentiation of olfactory progenitor cells, and the loss of their function prevents the normal differentiation of olfactory progenitor cells into mature olfactory neurons. ${ }^{11}$ Since then, more and more studies have found that the miR200 family participates in a variety of human diseases, especially in the occurrence and development of cancer. Gregory et al found that the miR-200 family regulates epithelialmesenchymal transition by targeting ZEB1 and SIP1, which is an important step for breast cancer infiltration and metastasis. ${ }^{12}$ Adam et al used miRNA microarray and realtime fluorescent quantitative PCR to screen and verify that the miR-200 family, abnormally expressed in bladder cancer, participates in the migration and EMT process of bladder cancer, and increases the sensitivity to EGFR blockers. ${ }^{13}$ In addition to breast cancer and bladder cancer, more and more evidences also show that the abnormal expression of the microRNA-200 family is closely related to the progression of hepatocellular carcinoma.

\section{Expression Changes of MiR-200 Family in HCC}

Studies on identifying miRNAs that are abnormally expressed in hepatocellular carcinoma provide important information for the role of miRNAs in tumor biology. Furthermore, researches focusing on the miRNA-200 family offer new insights for the exploration of the mechanisms, as well as the screening, diagnosis and prognosis monitoring of hepatocellular carcinoma. Even more interesting is that most studies demonstrate that the expressions of miR-141, miR-200a, miR-200b, miR-200c and miR-429 were down regulated in hepatocellular carcinoma. Back in 2008, analyzing of miRNA expression 
A

Cluster miR-200b/200a/429

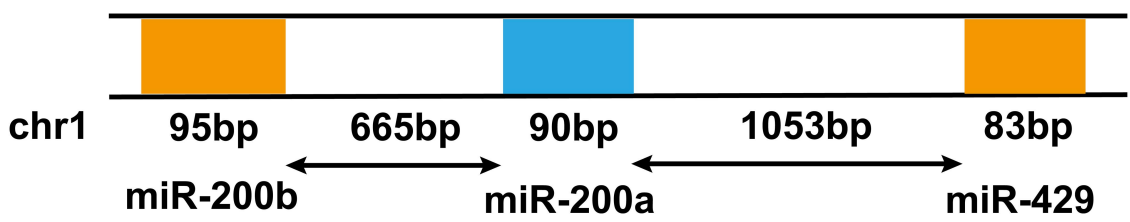

Cluster miR-200c/141

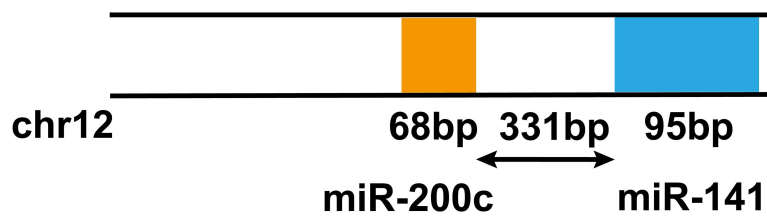

B

\begin{tabular}{|c|c|}
\hline $\begin{array}{l}\operatorname{miR}-141 \\
\operatorname{miR}-200 a\end{array}$ & $\begin{array}{l}\text { UAACEACUGUCUGGUAAAGAUGG } \\
\text { UAACEACUGUCUGGUAACGAUGU }\end{array}$ \\
\hline miR-429 & UAAUAACUGUCUGGUAAAACCGU \\
\hline miR-200b & UAAUACUGCCUGGUAAUGAUGA \\
\hline miR-200c & UAAUACUGCCGGGUAAUGAUGGA \\
\hline
\end{tabular}

Figure I The location on human chromosome and the sequence of the miR-200 family. (A) The miR-200 family can be divided into two clusters according to the location on the chromosome. The cluster, located on the human chromosome I, contains miR-200b, miR-200a and miR-429. The cluster, located on the human chromosome 12, consists of miR-200c and miR-I4I; (B) The miR-200 family can also be divided into two groups depending on the seed sequence (red color). The difference between the two groups is the third nucleotide of the seed sequence. The seed sequence of the group, containing miR-I4I and miR-200a, is AACACUG while the seed sequence of the group, including miR-429, miR-200b and miR-200c, is AAUACUG.

levels in 400 samples of more than 20 cancer tissues, Rosenfeld et al have found a low expression of miR-141 in primary $\mathrm{HCC}$ according to the miRNA microarray data. $^{14}$ Barshack et al have observed concentration of miR-200c in liver cancer tissue decreased significantly compared with normal liver tissue using gene chip and fluorescence quantitative PCR to detect the expression level of 250 miRNAs in 46 primary hepatocellular carcinoma tissues and 4 normal liver tissues. ${ }^{15}$ Studies have confirmed that the expression level of miR-200b in liver cancer tissues is significantly down regulated compared with adjacent tissues; compared with L02 cells, miR200 b expression in HepG2 cells was lower. ${ }^{16}$ Subsequent studies have also reported that the expression of miR-200a in the miR-200 family is down-regulated in liver cancer tissues and liver cancer cell lines. ${ }^{17}$ Guo et al used quantitative real-time fluorescence polymerase chain reaction (qRT-PCR) to detect miR-429 in 12 HCC and non-tumor liver tissues. And the results showed that the expression level of miR-429 in HCC tissues was significantly lower than that of the corresponding non-tumor liver organization. ${ }^{18}$ Wong et al have reported that five members of the miR-200 family were all down regulated in liver cancer tissues through the detection of miRNA expression profile in 20 cases of liver cancer tissues using heat maps. ${ }^{19}$ The above researches suggest that members of the miR-200 family have the potential to become biomarkers for the diagnosis of HCC.

At present, the views on changes in the level of miR200 family in the development of hepatocellular carcinoma are also generally consistent: the five members of the miR-200 family generally have a low expression pattern and serve as important tumor suppressors in hepatocellular carcinoma. On the contrary, a few studies showed some members of the miR-200 family were upregulated in hepatocellular carcinoma. In order to investigate the mechanisms of liver cancer infected by hepatitis $\mathrm{C}$ virus, Banaudha et al silenced miR-141 with an oligonucleotide complementary to miRNA, thereby inhibiting virus replication. Artificially increasing the level of miR-141 in the 
cell enhances the replication of HCV virus. Therefore, the researchers believe that the overexpression of miR-141 in the body is the basis for the replication of hepatitis $\mathrm{C}$ virus, thereby inducing the occurrence of liver cancer. ${ }^{20}$ Another study found that miR-429 expression increased in liver cancer tissues and was closely related to tumor size and aflatoxin DNA content by examining 138 cases of liver cancer tissues with pathological diagnosis. ${ }^{21}$ Similarly, another report investigated the levels of miR-429 in healthy liver tissues, chronic hepatitis B, liver cirrhosis and liver cancer tissues, and found that miR-429 was highly expressed in liver cancer tissues. Correlation regression analysis showed that high expression of miR429 in liver cancer was closely associated with a variety of malignant pathological features. ${ }^{22}$

We believe that the above-mentioned differences in the expression studies of the two members of the miR-200 family in liver cancer may be caused by the source of the samples, the number of samples, and different analysis methods. In addition, it is worth noting that the difference in hepatitis virus species may also be an important reason for the different expression of miR-200 family in liver cancer. In short, we still need further research. The research should be based on a large sample of data and use a more refined and reasonable grouping design to draw conclusions that are closer to facts.

\section{Molecular Mechanisms of MiR-200 Family in HCC}

Often, microRNA regulate the expression of genes and their downstream signaling pathways at the posttranscriptional level. At the same time, microRNA itself is also regulated by upstream molecules such as lncRNA. In recent years, more and more evidences have shown that a variety of IncRNAs play an important role in the malignant behavior of hepatocellular carcinoma by regulating the expression of the miR-200 family and further regulating downstream target genes and signaling pathways. Therefore, further understanding of the roles of the miR200 family and its upstream and downstream regulatory molecules in carcinogenesis may provide new insights into the mechanism of hepatocellular carcinoma development.

\section{MiR-200 Family and IncRNAs}

Long non-coding RNA (lncRNA) is a large group of ribonucleic acid transcripts with more than 200 nucleotides and no obvious open reading frame. ${ }^{23}$ It has a specific expression pattern during development or disease, and plays a decisive role in many pathophysiological processes, especially in tumors, although lncRNA cannot encode proteins. ${ }^{24-27}$ LncRNA can regulate cell proliferation, apoptosis, cycle, and even cancer cell migration and invasion by directly binding to protein, mRNA or microRNA. ${ }^{27}$ Yuan et al have found that activation of IncRNA-AL by transforming growth factor$\beta$ can mediate the migration and invasion of HCC cells via the TGF- $\beta$ /miR-200/ZEB signaling pathway. ${ }^{28}$ Subsequent studies have identified many abnormally expressed lncRNAs associated with the miR-200 family in HCC.

HULC is a key lncRNA abnormally expressed in HCC, whose high expression is closely related to the poor prognosis of patients with HCC, and located on mammalian chromosome 6q24.3. Studies have confirmed that miR200a is negatively correlated with HULC and ZEB1 expression in liver cancer using the Kaplan-Meier analysis and the Log rank test. ${ }^{29}$ As an endogenous competitive RNA, it can isolate the signal pathway of miR-200a and up-regulating ZEB1 to mediate the EMT process and promote the occurrence and development of HCC. Sui et al have identified a lncRNA that continues to increase in the development of HCC named GIHCG. ${ }^{30}$ The expression of GIHCG in HCC tissues was up-regulated compared with adjacent nontumor tissues, and the expression level of it was positively correlated with tumor size, microvascular invasion, BCLC grade, and prognosis of HCC patients. Further experiments found that GIHCG is closely related to EZH2 and miR$200 \mathrm{a} / \mathrm{miR}-200 \mathrm{~b} / \mathrm{miR}-429$. It can increase the proliferation and migration of liver cancer cells in vitro and promoting the development and metastasis of xenograft tumors in vivo by recruiting EZH2 and DNMT1 to the miR-200a/miR200b/miR-429 promoter region to up-regulate the promoter region DNA and histone methylation, and silence the expression level of miR-200a/miR-200b/miR-429.

In summary, lncRNA can interact with miR-200 members competitively participate in regulating the expression of miR-200 downstream target molecules and play a key role in the progression of HCC.

\section{MiR-200 Family and Target Genes}

MiRNAs display different biological functions after binding to target genes to regulate most genes in mammals. Therefore, most biological processes in the body involve miRNA and miRNA-mediated gene expression regulation.

The MiR-200 family has been reported to promote the growth of tumor cells and participate in the regulation of various cancers via regulating ZEB1 and ZEB2. ${ }^{31,32}$ ZEB1 
and ZEB2 are important members of the zinc finger enhancer-binding protein family. Studies have confirmed that miR-200a, miR-200b and ZEB-1 expression levels have a significant negative correlation, using immunohistochemistry and Spearman-Rho method to analyze the expression intensity of miR-200 family and ZEB- 1 in HCC. ${ }^{33} \mathrm{Li}$ et al have found that miR-200b can be combined with the 463-479 and 892-898 base positions in the ZEB1 mRNA 3'UTR through the analysis of bioinformatics software and have confirmed that ZEB1 is the target molecule of miR-200b, and miR-200b can binding mRNA of ZEB1 to inhibit its translation by the method of the luciferase reporter gene. ${ }^{34}$ It has also been reported that ZEB2 contains three potential sites capable of binding to miR-141, and further confirmed that ZEB2 is the target molecule of miR-141 and overexpression of ZEB2 can reverse inhibition of miR-141 on HCC cells by luciferase reporter gene and Western blot experiments. ${ }^{35}$ Another study confirmed that the high expression of $\mathrm{G \alpha} 12$ in HCC can promote the growth of cancer by releasing p53reactive miRNA and inducing the expression of ZEB1. ${ }^{36}$ The above research data shows that the ZEB1/ZEB2 axis in HCC is an important regulator of the entire miR-200 family.

B-cell-specific Moloney leukemia virus inset site 1 (BMVI 1) is a carcinogenic gene of various cancers, which is also highly expressed in HCC. ${ }^{37,38} \mathrm{Wu}$ et al used fluorescence quantitative PCR to detect the expression levels of miR-200b and BMI1 in liver cancer tissues and found that miR-200b expression was downregulated in $83.3 \%$ of cancers. ${ }^{39}$ BMI1 was significantly overexpressed in $66.7 \%$ of cancer tissues, indicating that the expression of BMI1 and miR-200b is negatively correlated in HCC. The luciferase reporter gene experiment further confirmed that BMI1 is the target molecule of miR$200 \mathrm{~b}$ and it can participate in the regulation of HCC.

In addition to the above genes, miR-200 can also target other genes (Figure 2). These reveal that the target genes regulated by the miR-200 family in HCC are complex and diverse. In HCC, it needs further discussion on which target gene plays a leading role in the occurrence and development of cancer, or which family member of miR200 can specifically regulate this target gene.

\section{MiR-200 Family and Target Gene Downstream Signaling Pathway}

The miR-200 family can regulate the occurrence and development of HCC through a variety of downstream signaling pathways, and the dysregulation of signaling pathways will lead to the continuous deterioration of HCC. Phosphatidylinositol-3-kinase/threonine kinase (PI3K/AKT) can control the cycle, apoptosis, metabolism and molecular mutations of cancer cells, which is one of the most critical molecular conduction pathways involved in the process of cell life activities. ${ }^{40}$ An investigation

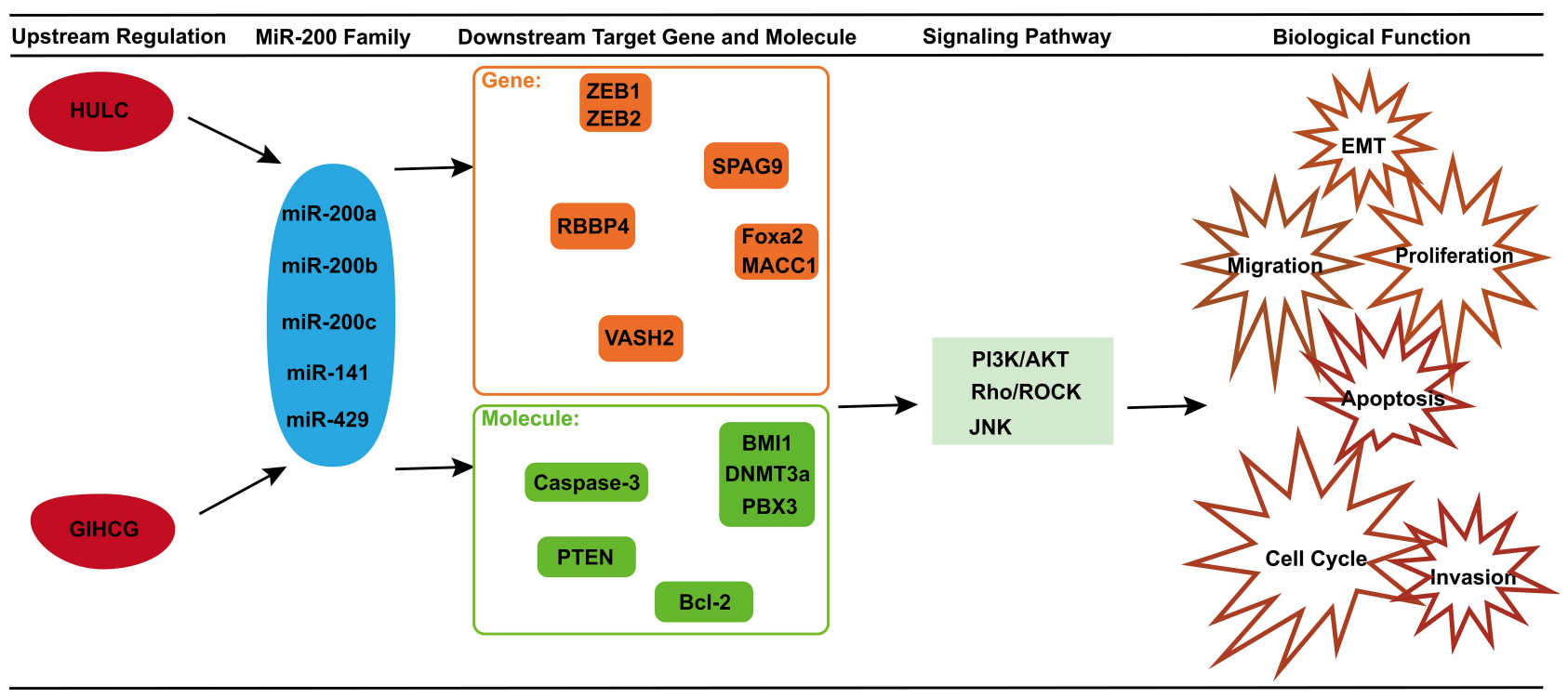

Figure 2 Related molecules, genes, signaling pathways and biological function in HCC of miR-200 family. Two IncRNAs, HULC and GIHCG, can inhibit the miR-200 family. The miR-200 family can target ZEBI, ZEB2, SPAG9, RBBP4, Foxa2, MACCI and VASH2. And they can regulate Caspase-3, BMII, DNMT3a, PBX3, PTEN and Bcl-2. Finally, through PI3K/AKT, Rho/ROCK or JNK signaling pathway, the miR-200 family can mediate many tumor cell biological functions, like EMT, migration, invasion, proliferation and apoptosis. 
proved that miR-429 can target the tumor suppressor molecule PTEN to activate the classic PI3K/AKT pathway. It increases the metastatic ability of HCC cells by inducing the phosphorylation of AKT to inhibit the activity of GSK-3 $\beta$ and $\beta$-catenin. ${ }^{41}$

Rho is a member of the Ras superfamily with a molecular weight of $20-30 \mathrm{kDa}$. It is affected by many factors and plays an important role in the molecular network. As an important downstream effector of Rho, Rhorelated kinase (ROCK) mediates the migration of cancer cells and vascular remodeling by regulating the adhesion of actin filament cytoskeleton and lesions. ${ }^{42,43}$ Wong et al found that the miR-200b/miR-200c/miR-429 subfamily can inhibit the metastasis of liver cancer by targeting the Rho/ROCK signaling pathway to reorganize the cytoskeleton and reduce the adhesion of the basement membrane. ${ }^{19}$

c-Jun N-terminal kinases (JNKs) are important members of the MAPK system. Named for its ability to phosphorylate and activate protein c-Jun, JNK can play a central role in various cancer cell responses. New evidence shows that in HCC cell lines over-expressed with miR-141, the JNK activity of the downstream signaling pathway of the target gene SPAG9 can be silenced, the expression of related molecules c-Jun and MMP9 in the JNK signaling pathway is down-regulated, and restoration of SPAG9 expression can significantly reverse the inhibition of miR-141 on the activity of JNK signaling pathway. ${ }^{44}$

Related studies have shown that the miR-200 family affects various processes of HCC cells by being involved in different signaling pathways. Although different members can target many signaling pathways individually, the signaling pathways dominated by various gene clusters in HCC are also different. This theory can lay a solid foundation for miR-200 members to use for individualized treatment and comprehensive treatment of HCC.

\section{The Biological Function of MiR-200 Family in the Process of HCC}

It is clearly found that miRNAs in organisms not only regulate downstream target genes and multiple signal transduction pathways at the post-transcriptional level, but their expression are affected by upstream molecules. The regulatory relationship between them may be related to each other through complex linear relationships such as point-to-many or multi-point to participate in the regulation of EMT, cell proliferation, cell apoptosis, cell cycle, cell migration, cell invasion and other biological processes. As mentioned above, the abnormal expression of miR-200 family is closely related to the occurrence and development of hepatocellular carcinoma. At the same time, a large number of studies have found that cell functions can also be changed by regulating the upstream and downstream molecules of miR-200 family in hepatocellular carcinoma.

\section{MiR-200 Family and EMT}

Epithelial-mesenchymal transition (EMT), the process of transforming epithelial components into mesenchymal components, is considered to be a key step in the process of cancer invasion and migration. During the occurrence of this process, the basal polarity, tight connectivity, and adhesion of epithelial cells are gradually lost, resulting in an increase in mesenchymal cells and fibroblasts, which is beneficial to the migration of tumor cells. EMT mainly activates genes of the mesenchymal related phenotype by transcription factors such as ZEB, SNAIL, AKT2 and FOX, and further inhibits epithelial marker genes to activate the epithelial-stromal process. These transcription factors can bind to the promoter region of EMT molecules and lead to the inactivation of transcription of epithelial markers such as E-cadherin and the activation of mesenchymal molecules N-cadherin and Vimentin. Multiple studies have shown that EMT plays an important role in various tumors such as liver, breast and prostate. ${ }^{45-47}$

The MiR-200 family is first discovered and also the most extensively studied EMT-related miRNA family. ${ }^{48}$ Wong et al found that the levels of miR-200 family and E-cadherin in liver cancer cell lines are oppositely related. ${ }^{19}$ Besides, studies have reported that in MHCC97L, miR-200a can suppress the EMT process in vitro via regulating the transcription and post-transcription levels of Vimentin and E-cadherin. ${ }^{49}$ Yang et al found that miR200a can bind to the ZEB2 promoter region to increase the expression of E-cadherin protein and reduce the expression of N-cadherin protein and thereby mediate the EMT process of hepatoma side population cells. ${ }^{50}$ In addition, another study found that miR-141 could reverse the EMT process by targeting GP73, and subsequently inhibited the progression and metastasis of HCC. ${ }^{51}$ Therefore, the study of the specific molecular mechanism of miR-200 family mediating the EMT process plays an important role in the development of effective targets for the treatment of HCC. 


\section{MiR-200 Family and Cell Proliferation}

The MiR-200 family can inhibit the proliferation of tumor cells. Zhong et al found that silencing miR-200a in HCC cell line MHCC-97L can significantly increase the proliferation ability of cells. ${ }^{49}$ Further research confirmed that overexpression of miR-200a can significantly inhibit the proliferation activity and colony formation rate of HCCLM3 and HepG2 cells while knocking out miR200a can restore the proliferation ability of liver cancer cells and increase the rate of colony formation. ${ }^{52}$ Also, there are studies have found that miR-141 can target HNF$3 \beta$ to inhibit the growth rate of HepG2 cells and overexpression of HNF-3 $\beta$ can partially relieve the inhibitory effect of miR-141 by using pre-miR-141, HNF-3 $\beta$ siRNA or HNF- $3 \beta$ plasmid to transfect HepG2 to construct stable cell lines. ${ }^{53}$ A study on miR-200b/200a/429 found that GHICG physically combined with the promoter of miR$200 \mathrm{~b} / 200 \mathrm{a} / 429$ to significantly silence the expression of miR-200b/200a/429, thereby inhibiting the proliferation and migration of liver cancer cells, which provides new insights into the functions of miR-200b/200a/429 in liver cancer and helps to understand the pathophysiological process of HCC-related diseases. ${ }^{30}$ Similarly, Li et al found that the low expression of miR-200c-5p in liver cancer tissues and cells is closely related to the significant overexpression of MAD2L1 compared with control group. Up-regulation of miR-200c-5p reduced the expression of MAD2L1, and inhibited the proliferation, migration, invasion of HCC cells, and induced their apoptosis and cell cycle arrest. ${ }^{54}$ The above experiments provide important theoretical support for the miR-200 family to inhibit the growth of HCC.

\section{MiR-200 Family and Apoptosis, Cell Cycle}

Apoptosis, as programmed cell death, is an important way for the body to maintain normal growth and development. Anti-apoptotic signaling is common to tumor cells and this can lead to the development of multiple drug resistance.

Bcl-2 family can mediate apoptosis pathway. ${ }^{55}$ It has been reported that miR-200b in the miR-200 family can negatively regulate the expression level of Bcl-2 in HCC leading to an increase in the rate of apoptosis. ${ }^{39}$ In addition, miR-200a can also increase the apoptosis rate of MHCC-97L by regulating Caspase- $3 .{ }^{49}$ Lin et al found that the miR-141 group's apoptosis rate increased significantly compared with the control group. ${ }^{56}$ In addition, there are related reports on the research that miR-200c and miR-429 may regulate the apoptosis of liver cancer cells. ${ }^{54,57}$ Comprehensive analysis of the miR-200 family's regulation of apoptosis can provide new ideas for the clinical treatment of HCC.

The cell cycle is an important process for the body to grow and reproduce. Cell cycle-dependent protein kinases can regulate the cell cycle process and abnormal regulators of the coding cycle can lead to the occurrence of a variety of cancers. MiRNA can suppress the occurrence of HCC by adjusting the redistribution of the cell cycle. By transfecting miR-200b mimics, Wu et al found that HCC cells stopped in the G1 phase, which reducing the number of $\mathrm{S}$ and G2 phases and resulting in changes in cell cycle distribution. ${ }^{39}$ Studies on miR-200a and miR-200c found that microRNA-200a and miR-200c were independent prognostic factors for hepatocellular carcinoma, and induced cell cycle arrest by targeting CDK6 or MAD2L1 respectively. ${ }^{54,58}$ However, there are few reports about the miR-200 family regulating the liver cancer cell cycle. Whether other members of the miR-200 family can affect the progression of hepatocellular carcinoma by regulating cyclin in vivo requires further investigation.

\section{MiR-200 Family and Cell Migration, Cell Invasion}

The migration and invasion ability of cancer cells is extremely important for the treatment and prognosis of cancer patients. High metastasis, high invasiveness and high recurrence are the main reasons for the high mortality and low survival rate of HCC patients. As an important inhibitor molecule, the miR-200 family is closely related to the migration and invasion of liver cancer.

A recent investigation report confirmed that miR-200a expression was down-regulated in tissues and cell lines of liver cancer, and the overexpression of miR-200a can inhibit the migration and invasion of liver cancer cells in vitro by targeting GAB1 molecules, while GAB1 restoration may partially alleviate the inhibitory effect of miR-200a. ${ }^{17}$ In addition, miR-200b can also target ZEB1 and BMI1 resulting in low expression of these molecules to inhibit the migration and invasion of HCC cells. ${ }^{34,38}$ Reports on miR-141 mediating the migration and invasion of HCC cells show that up-regulation of miR-141 can inhibit the migration and invasion of HCC cells while knocking out miR-141 increases the migration and invasion capacity of cells. ${ }^{35,44}$ The change of EZH2 gene copy number status induces BMI1-mediated migration and 
invasion of liver cancer cells by epigenetic silencing miR$200 \mathrm{c}$, which plays a key role in the occurrence and development of liver cancer. $^{59}$

The above studies have clarified the specific mechanism of the miR-200 family in the invasion and migration of HCC cells and provide a solid theoretical basis for further exploring the molecular mechanism of the miR-200 family to inhibit the migration and invasion of cancer cells.In general, the miR-200 family, as an important regulator of tumors, has a significant impact on the development of HCC in most cases. It participates in the regulation of various biological functions of HCC (Table 1). This provides a better basis to find new biomarkers for the clinical treatment of $\mathrm{HCC}$.

\section{The Clinical Value of MiR-200 Family in HCC}

Above all, a large number of studies on hepatocellular carcinoma have shown that the miR-200 family participates in regulating the malignant biological behavior of tumors by regulating the expression of target genes, which may become an important marker for the diagnosis and prognosis of hepatocellular carcinoma, and may even be used as an anti-tumor aid therapy.

\section{MiR-200 Family and Early Diagnosis and Prognostic Monitoring of HCC}

The late diagnosis of HCC is accompanied by the patient's poor prognosis and low survival rate. Therefore, there is an urgent clinical need to find specific biological markers to make an early diagnosis of HCC. Researches have confirmed that the miR-200 family is extremely stable in cells and is considered a potential non-invasive candidate for cancer diagnosis. ${ }^{60,61}$ Retrospectively tracking a set of serum miRNAs of liver cancer patients in 7 medical centers in China, Li et al found that miR-429 had the best AUC value of 0.670 , with sensitivity and specificity of $35.4 \%$ and $97.1 \%$; while AUS of AFP was 0.603 , sensitivity was $36.9 \%$, and specificity was $83.8 \%{ }^{62}$ Although miR-429 showed the best screening value in this study, due to factors such as racial differences and sample size limitations, the researchers believe that only the combined

Table I Functions That the miR-200 Family Involved in HCC

\begin{tabular}{|c|c|c|}
\hline Functions & Related miR-200 Family Members & Reference \\
\hline EMT & $\begin{array}{l}\mathrm{miR}-200 \mathrm{~b} / 200 \mathrm{c} / 429 \\
\mathrm{miR}-200 \mathrm{a} \\
\mathrm{miR}-200 \mathrm{a} \\
\mathrm{miR}-|4|\end{array}$ & $\begin{array}{l}\text { Wong et al }(2015)^{19} \\
\text { Zhong et al }(2015)^{49} \\
\text { Yang et al }(2015)^{50} \\
\text { Hou et al }(2019)^{51}\end{array}$ \\
\hline Cell Proliferation & $\begin{array}{l}\text { miR-200a } \\
\text { miR-200a } \\
\text { miR-14l } \\
\text { miR-200b/200a/429 } \\
\text { miR-200c }\end{array}$ & $\begin{array}{l}\text { Zhong et al }(2015)^{49} \\
\text { Feng et al }(2015)^{52} \\
\text { Lou et al }(2016)^{44} \\
\text { Sui et al }(2016)^{30} \\
\text { Li et al }(2017)^{54}\end{array}$ \\
\hline Cell Apoptosis & $\begin{array}{l}\text { miR-200b } \\
\text { miR-200a } \\
\text { miR-14I } \\
\text { miR-200c } \\
\text { miR-429 }\end{array}$ & $\begin{array}{l}\text { Wu et al }(2016)^{39} \\
\text { Zhong et al }(2015)^{49} \\
\text { Lin et al }(2014)^{56} \\
\text { Li et al }(2017)^{54} \\
\text { Gao et al }(2014)^{57}\end{array}$ \\
\hline Cell Cycle & $\begin{array}{l}\text { miR-200a } \\
\text { miR-200b } \\
\text { miR-200c }\end{array}$ & $\begin{array}{l}\text { Xiao et al }(2013)^{58} \\
\text { Wu et al }(2016)^{39} \\
\text { Li et al }(2017)^{54}\end{array}$ \\
\hline Metastasis and Invasion & $\begin{array}{l}\text { miR-200a } \\
\text { miR-200b } \\
\text { miR-200b } \\
\text { miR-I4I } \\
\text { miR-14I } \\
\text { miR-200c }\end{array}$ & $\begin{array}{l}\text { Wang et al }(2017)^{17} \\
\text { Li et al }(2016)^{34} \\
\text { Effendi K et al }(2010)^{38} \\
\text { Wu et al }(2014)^{35} \\
\text { Lou et al }(2016)^{44} \\
\text { Xu et al }(2020)^{59}\end{array}$ \\
\hline
\end{tabular}

Abbreviation: EMT, epithelial-mesenchymal transition. 
application of three methods, such as miR-429, serum miRNA, alpha-fetoprotein, and ultrasound, can optimize the effectiveness of early screening and prognostic monitoring of high-risk populations of HCC. The results of this study also show that serum miRNA is a kind of noninvasive biomarker that worth promoted.

Postoperative follow-up of cancer patients is extremely important to improve the long-term survival rate and prognosis of cancer patients. But according to the existing biological detection technology, it is impossible to make a long-term prediction and real-time monitoring of the postoperative condition of cancer patients. Therefore, finding a biological marker with high specificity and convenient detection is a strong demand of contemporary medical practice. Feng et al have reported that the degree of vascular invasion, pre-operative expression of alphafetoprotein, tumor size, and miR-200a expression level were all predictors of overall survival and cumulative recurrence of $\mathrm{HCC}$ by using univariate analysis. Patients with low expression of miR-200a have a worse prognosis than those with high expression of miR-200a, was increased cumulative recurrence rates at 1,3 and 5 years, and reduced survival rates at 1,3 and 5 years. ${ }^{52}$ Karakatsanis et al have found that the expression of miR-200c in HCC is down-regulated and closely related to clinical-pathological factors and prognostic survival rate through examining the expression of miRNA in non-tumor liver and HCC. ${ }^{63}$ Yao et al conducted a follow-up survey and found that patients in the low-expression miR-141 group had a lower 3-year survival rate than those in the high-expression miR-141 group. $^{64}$ A study conducting Kaplan-Meier analysis on $161 \mathrm{HCC}$ tissue samples has found that miR-429 expression level is negatively correlated with patient survival rate. ${ }^{22}$

MiR-200 in circulating blood is expected to become a specific biomarker for early diagnosis and prognosis prediction of HCC in the future. To achieve this goal, we also need to solve the following problems: first, establish a perfect miR-200 expression system in animal models. Second, collect well-characterized patient sera as paired samples. Third, reduce expensive and time-consuming miRNA detection methods.

\section{MiR-200 Family as Therapeutic Target}

The main strategy of hepatocellular carcinoma treatment is to combine surgery with chemotherapy, and the main reason for the failure of diagnosis and treatment of hepatocellular carcinoma is often the development of chemotherapy resistance. A large number of research results show that the main reasons for cancer resistance to specific drugs are the increase in anti-cancer drug detoxification, the inactivation of apoptotic pathways, changes in DNA repair or DNA tolerance, and the decrease in the intake of water-soluble drugs. ${ }^{65}$ More and more evidence shows that the miR-200 family plays an important role in regulating the drug resistance of hepatocellular carcinoma treatment. 5-fluorouracil is a chemical drug used to treat hepatocellular carcinoma. The study by Shi et al found that overexpression of miR141 in HepG2 could lead to the restoration of miR-141 and the inhibition of target genes, ultimately resulting in the enhancement of 5-fluorouracil resistance, while transfection of miR-141 inhibitors can partially restore the toxicity and apoptosis induced by the molecule, which indicates that miR-141 plays a key role in the 5-fluorouracil treatment of liver cancer resistance. ${ }^{66}$ One study was analyzed by MTT, flow cytometry cell cycle analysis and TUNEL analysis of curcumin against J5-200a, J5-200b and J5-control to evaluate the anti-cancer effect of cells. The results demonstrated that the expression level of miR$200 \mathrm{a} / \mathrm{b}$ determines the therapeutic efficacy of curcumin on HCC cells. ${ }^{67}$ In this case, miRNAs that predict the treatment response of a given patient may help clinicians choose the right treatment.

Due to disorders in cancer, miRNAs are generally divided into two types. One is tumor suppressor miRNA, and the other is acting as an oncogene. According to the two unique functions of miRNA in cancer, an innovative therapy based on miRNA has emerged. Many preclinical trials have shown that miRNAs affect the sensitivity of tumors to traditional anti-tumor therapies by using effective delivery strategies (such as chemical modification, virus-based vectors, non-viral lipid carriers and exosomes). ${ }^{68}$ As an indepth study of tumor suppressors, the miR-200 family is definitely a suitable candidate drug for the treatment of hepatocellular carcinoma. A study on miRNA as a drug for in vivo delivery found that compared with the negative control, a tissue-adhesive nucleotide-polymer complex (NPX-gel) coated with miR-141 was injected into tumors in mouse significantly inhibited the growth of tumors, so as to achieve the effect of local treatment of hepatocellular carcinoma. ${ }^{69}$ An effective delivery vector provides the possibility for the miR-200 family to overcome many extraand intracellular barriers of hepatocellular carcinoma cells. This is the guarantee of the miR-200 family as a therapeutic target to play an anti-tumor effect. 
However, there are currently few reports on the research of other members of the miR-200 family as therapeutic targets for hepatocellular carcinoma. Related research needs to be further explored in order to truly apply the miR-200 family therapy in clinical practice.

\section{Conclusion and Outlook}

The miR-200 family is a key regulator of liver cancer development. It can regulate cell proliferation, apoptosis, cycle distribution and participate in tumor invasion, migration, and chemoresistance. It promotes the EMT process of cancer. Because of the above, the miR-200 family is expected to become a new target and provides new opportunities for further molecular treatment of HCC. As research continues, the mystery of the miR-200 family's role in liver cancer will slowly unravel. However, the specific role of genes in tumors is based on tumor heterogeneity and individual differences. To further clarify these molecular mechanisms, the current research focus is mainly on the specific functions of individual miRNA200 family members. If relating the difference in expression of miRNA-200 family members to specific different functions in liver tumor background, it will contribute to the discovery of new mechanisms of miRNA-200 family in HCC progression. MiRNA can affect multiple target molecules and participate in the entire interacting molecular network system. The effect it produces is a doubleedged sword. How to transform basic research results into real medical practice safely and effectively needs to explore and trail.

\section{Acknowledgments}

This work was supported by the National Key Research and Development Plan of China (2018YFC2000200), National Natural Science Foundation of China (81772673), Shanghai Sailing Program (19YF1405500) and the Initial Scientific Research Fund of Huashan Hospital Affiliated to Fudan University (2019QD003).

\section{Disclosure}

The authors report no conflicts of interest in this work.

\section{References}

1. Marquardt JU, Andersen JB, Thorgeirsson SS. Functional and genetic deconstruction of the cellular origin in liver cancer. Nat Rev Cancer. 2015;15(11):653-667. doi:10.1038/nrc4017

2. Maluccio M, Covey A. Recent progress in understanding, diagnosing, and treating hepatocellular carcinoma. CA Cancer J Clin. 2012;62 (6):394-399. doi:10.3322/caac.21161
3. Bracken CP, Scott HS, Goodall GJ. A network-biology perspective of microRNA function and dysfunction in cancer. Nat Rev Genet. 2016;17:719-732. doi:10.1038/nrg.2016.134

4. Friedman RC, Farh KK, Burge CB, Bartel DP. Most mammalian mRNAs are conserved targets of microRNAs. Genome Res. 2009;19:92-105. doi:10.1101/gr.082701.108

5. Lin S, Gregory RI. MicroRNA biogenesis pathways in cancer. Nat Rev Cancer. 2015;15:321-333. doi:10.1038/nrc3932

6. Mavrakis KJ, Van Der Meulen J, Wolfe AL, et al. A cooperative microRNA-tumor suppressor gene network in acute T-cell lymphoblastic leukemia (T-ALL). Nat Genet. 2011;43:673-678. doi:10.1038/ ng. 858

7. Song SJ, Poliseno L, Song MS, et al. MicroRNA-antagonism regulates breast cancer stemness and metastasis via TET-familydependent chromatin remodeling. Cell. 2013;154:311-324. doi:10.1016/j.cell.2013.06.026

8. Calin GA, Croce CM. MicroRNA signatures in human cancers. Nat Rev Cancer. 2006;6:857-866. doi:10.1038/nrc1997

9. Paterson EL, Kazenwadel J, Bert AG, Khew-Goodall Y, Ruszkiewicz A, Goodall GJ. Down-regulation of the miRNA-200 family at the invasive front of colorectal cancers with degraded basement membrane indicates EMT is involved in cancer progression. Neoplasia. 2013;15:180-191. doi:10.1593/neo.121828

10. Humphries B, Yang C. The microRNA-200 family: small molecules with novel roles in cancer development, progression and therapy. Oncotarget. 2015;6(9):6472-6498. doi:10.18632/oncotarget.3052

11. Choi PS, Zakhary L, Choi W-Y, et al. Members of the miRNA-200 family regulate olfactory neurogenesis. Neuron. 2008;57(1):41-55. doi:10.1016/j.neuron.2007.11.018

12. Gregory PA, Bert AG, Paterson EL, et al. The miR-200 family and miR-205 regulate epithelial to mesenchymal transition by targeting ZEB1 and SIP1. Nat Cell Biol. 2008;10(5):593-601. doi:10.1038/ ncb1722

13. Adam L, Zhong M, Choi W, et al. miR-200 expression regulates epithelial-to-mesenchymal transition in bladder cancer cells and reverses resistance to epidermal growth factor receptor therapy. Clin Cancer Res. 2009;15(16):5060-5072. doi:10.1158/1078-0432.Ccr-08-2245

14. Rosenfeld N, Aharonov R, Meiri E, et al. MicroRNAs accurately identify cancer tissue origin. Nat Biotechnol. 2008;26(4):462-469. doi:10.1038/nbt1392

15. Barshack I, Meiri E, Rosenwald S, et al. Differential diagnosis of hepatocellular carcinoma from metastatic tumors in the liver using microRNA expression. Int $J$ Biochem Cell Biol. 2010;42 (8):1355-1362. doi:10.1016/j.biocel.2009.02.021

16. Li X-Y, Feng X-Z, Tang J-Z, et al. MicroRNA-200b inhibits the proliferation of hepatocellular carcinoma by targeting DNA methyltransferase 3a. Mol Med Rep. 2016;13(5):3929-3935. doi:10.3892/ mmr.2016.4995

17. Wang J, Song W, Shen W, et al. MicroRNA-200a Suppresses Cell Invasion and Migration by Directly Targeting GAB1 in Hepatocellular Carcinoma. Oncol Res Featur Preclin Clin Cancer Therapeutics. 2017;25(1):1-10. doi:10.3727/096504016x1468 5034103798

18. Guo C, Zhao D, Zhang Q, Liu S, Sun MZ. miR-429 suppresses tumor migration and invasion by targeting CRKL in hepatocellular carcinoma via inhibiting Raf/MEK/ERK pathway and epithelial-mesenchymal transition. Sci Rep. 2018;8:2375. doi:10.1038/s41598-018-20258-8

19. Wong C-M, Wei L, Au SL-K, et al. MiR-200b/200c/429 subfamily negatively regulates Rho/ROCK signaling pathway to suppress hepatocellular carcinoma metastasis. Oncotarget. 2015;6 (15):13658-13670. doi:10.18632/oncotarget.3700

20. Banaudha K, Kaliszewski M, Korolnek T, et al. MicroRNA silencing of tumor suppressor DLC-1 promotes efficient hepatitis C virus replication in primary human hepatocytes. Hepatology. 2011;53 (1):53-61. doi:10.1002/hep.24016 
21. Huang X-Y, Yao J-G, Huang H-D, et al. MicroRNA-429 Modulates Hepatocellular Carcinoma Prognosis and Tumorigenesis. Gastroenterol Res Pract. 2013;2013:804128. doi:10.1155/2013/804128

22. Li L, Tang J, Zhang B, et al. Epigenetic modification of MiR-429 promotes liver tumour-initiating cell properties by targeting Rb binding protein 4. Gut. 2015;64(1):156-167. doi:10.1136/gutjnl-2013305715

23. Fatica A, Bozzoni I. Long non-coding RNAs: new players in cell differentiation and development. Nat Rev Genet. 2014;15(1):7-21. doi: $10.1038 / \mathrm{nrg} 3606$

24. Batista PJ, Chang HY. Long noncoding RNAs: cellular address codes in development and disease. Cell. 2013;152(6):1298-1307. doi:10.1016/j.cell.2013.02.012

25. Guo H, Ahmed M, Zhang F, et al. Modulation of long noncoding RNAs by risk SNPs underlying genetic predispositions to prostate cancer. Nat Genet. 2008;26(10):1142-1150. doi:10.1038/nbt1392

26. Sun M, Kraus WL. From discovery to function: the expanding roles of long noncoding RNAs in physiology and disease. Endocr Rev. 2015;36:25-64. doi:10.1210/er.2014-1034

27. Schmitt AM, Chang HY. Long Noncoding RNAs in Cancer Pathways. Cancer Cell. 2016;29:452-463. doi:10.1016/j. ccell.2016.03.010

28. Yuan JH, Yang F, Wang F, et al. A long noncoding RNA activated by TGF- $\beta$ promotes the invasion-metastasis cascade in hepatocellular carcinoma. Cancer Cell. 2014;25:666-681. doi:10.1016/j. ccr.2014.03.010

29. Li SP, Xu HX, Yu Y, et al. LncRNA HULC enhances epithelial-mesenchymal transition to promote tumorigenesis and metastasis of hepatocellular carcinoma via the miR-200a-3p/ZEB1 signaling pathway. Oncotarget. 2016;7:42431-42446. doi:10.18632/ oncotarget.9883

30. Sui CJ, Zhou YM, Shen WF, et al. Long noncoding RNA GIHCG promotes hepatocellular carcinoma progression through epigenetically regulating miR-200b/a/429. J Mol Med. 2016;94:1281-1296. doi:10.1007/s00109-016-1442-z

31. Korpal M, Lee ES, Hu G, Kang Y. The miR-200 family inhibits epithelial-mesenchymal transition and cancer cell migration by direct targeting of E-cadherin transcriptional repressors ZEB1 and ZEB2. J Biol Chem. 2008;283:14910-14914. doi:10.1074/jbc.C800074200

32. Mitchell PS, Parkin RK, Kroh EM, et al. Circulating microRNAs as stable blood-based markers for cancer detection. Proc Natl Acad Sci U S A. 2008;105:10513-10518. doi:10.1073/pnas.0804549105

33. Dhayat SA, Mardin WA, Köhler G, et al. The microRNA-200 family-a potential diagnostic marker in hepatocellular carcinoma? J Surg Oncol. 2014;110:430-438. doi:10.1002/jso.23668

34. Li Q, Song W, Wang W, et al. Suppression of epithelial-mesenchymal transition in hepatocellular carcinoma cells by Krüppel-like factor 4 . Oncotarget. 2016;7:29749-29760. doi:10.18632/oncotarget.8831

35. Wu SM, Ai HW, Zhang DY, et al. MiR-141 targets ZEB2 to suppress HCC progression. Tumour Biol. 2014;35:9993-9997. doi:10.1007/ s13277-014-2299-9

36. Yang YM, Lee WH, Lee CG, et al. Ga12 gep oncogene deregulation of p53-responsive microRNAs promotes epithelial-mesenchymal transition of hepatocellular carcinoma. Oncogene. 2015;34:2910-2921. doi:10.1038/onc.2014.218

37. Siddique HR, Saleem M. Role of BMI1, a stem cell factor, in cancer recurrence and chemoresistance: preclinical and clinical evidences. Stem Cells. 2012;30:372-378. doi:10.1002/stem.1035

38. Effendi K, Mori T, Komuta M, Masugi Y, Du W, Sakamoto M. Bmi-1 gene is upregulated in early-stage hepatocellular carcinoma and correlates with ATP-binding cassette transporter B1 expression. Cancer Sci. 2010;101:666-672. doi:10.1111/j.1349-7006.2009.01431.x

39. Wu WR, Sun H, Zhang R, et al. Methylation-associated silencing of miR-200b facilitates human hepatocellular carcinoma progression by directly targeting BMI1. Oncotarget. 2016;7:18684-18693. doi:10.18632/oncotarget.7629
40. Li X, Wu C, Chen N, et al. PI3K/Akt/mTOR signaling pathway and targeted therapy for glioblastoma. Oncotarget. 2016;7:33440-33450. doi:10.18632/oncotarget.7961

41. Tang J, Li L, Huang W, et al. MiR-429 increases the metastatic capability of HCC via regulating classic Wnt pathway rather than epithelial-mesenchymal transition. Cancer Lett. 2015;364:33-43. doi:10.1016/j.canlet.2015.04.023

42. Chen ZY, Yao WJ. [Role of Rho/ROCK in the migration of vascular smooth muscle cells]. Sheng Li Ke Xue Jin Zhan. 2013;44:269-274.

43. Pranatharthi A, Ross C, Srivastava S. Cancer Stem Cells and Radioresistance: rho/ROCK Pathway Plea Attention. Stem Cells Int. 2016;2016:5785786. doi:10.1155/2016/5785786

44. Lou G, Dong X, Xia C, et al. Direct targeting sperm-associated antigen 9 by miR-141 influences hepatocellular carcinoma cell growth and metastasis via JNK pathway. $J$ Exp Clin Cancer Res. 2016;35:14. doi:10.1186/s13046-016-0289-z

45. Huang M, Wu S, Hu Q, et al. Agkihpin, a novel SVAE may inhibit the migration and invasion of liver cancer cells associated with the inversion of EMT induced by Wnt/ $\beta$-catenin signaling inhibition. Biochem Biophys Res Commun. 2016;479:283-289. doi:10.1016/j. bbrc.2016.09.060

46. De Craene B, Berx G. Regulatory networks defining EMT during cancer initiation and progression. Nat Rev Cancer. 2013;13:97-110. doi: $10.1038 / \mathrm{nrc} 3447$

47. Lin G, Gai R, Chen Z, et al. The dual PI3K/mTOR inhibitor NVPBEZ235 prevents epithelial-mesenchymal transition induced by hypoxia and TGF- $\beta 1$. Eur $J$ Pharmacol. 2014;729:45-53. doi:10.1016/j.ejphar.2014.02.011

48. Korpal M, Kang Y. The emerging role of miR-200 family of microRNAs in epithelial-mesenchymal transition and cancer metastasis. RNA Biol. 2008;5:115-119. doi:10.4161/rna.5.3.6558

49. Zhong C, Li MY, Chen ZY, et al. MicroRNA-200a inhibits epithelial-mesenchymal transition in human hepatocellular carcinoma cell line. Int J Clin Exp Pathol. 2015;8:9922-9931.

50. Yang X, Wang J, Qu S, et al. MicroRNA-200a suppresses metastatic potential of side population cells in human hepatocellular carcinoma by decreasing ZEB2. Oncotarget. 2015;6:7918-7929. doi:10.18632/ oncotarget. 3486

51. Hou X, Yang L, Jiang X, et al. Role of microRNA-141-3p in the progression and metastasis of hepatocellular carcinoma cell. Int $J$ Biol Macromol. 2019;128:331-339. doi:10.1016/j. ijbiomac.2019.01.144

52. Feng J, Wang J, Chen M, et al. miR-200a suppresses cell growth and migration by targeting MACC1 and predicts prognosis in hepatocellular carcinoma. Oncol Rep. 2015;33:713-720. doi:10.3892/or.2014.3642

53. Han H, Du Y, Zhao W, et al. Author Correction: PBX3 is targeted by multiple miRNAs and is essential for liver tumour-initiating cells. Nat Commun. 2019;10:2259. doi:10.1038/s41467-019-10052-z

54. Li Y, Bai W, Zhang J. MiR-200c-5p suppresses proliferation and metastasis of human hepatocellular carcinoma (HCC) via suppressing MAD2L1. Biomed Pharm. 2017;92:1038-1044. doi:10.1016/j. biopha.2017.05.092

55. Czabotar PE, Lessene G, Strasser A, Adams JM. Control of apoptosis by the BCL-2 protein family: implications for physiology and therapy. Nat Rev Mol Cell Biol. 2014;15:49-63. doi:10.1038/nrm3722

56. Lin L, Liang H, Wang Y, et al. microRNA-141 inhibits cell proliferation and invasion and promotes apoptosis by targeting hepatocyte nuclear factor-3 $\beta$ in hepatocellular carcinoma cells. BMC Cancer. 2014;14:879. doi:10.1186/1471-2407-14-879

57. Gao H, Liu C. miR-429 represses cell proliferation and induces apoptosis in HBV-related HCC. Biomed Pharm. 2014;68:943-949. doi:10.1016/j.biopha.2014.09.005

58. Xiao F, Zhang W, Zhou L, et al. microRNA-200a is an independent prognostic factor of hepatocellular carcinoma and induces cell cycle arrest by targeting CDK6. Oncol Rep. 2013;30:2203-2210. doi:10.3892/or.2013.2715 
59. Xu L, Lin J, Deng W, et al. EZH2 facilitates BMI1-dependent hepatocarcinogenesis through epigenetically silencing microRNA-200c. Oncogenesis. 2020;9:101. doi:10.1038/s41389-020-00284-w

60. Chen L, Chu F, Cao Y, Shao J, Wang F. Serum miR-182 and miR-331-3p as diagnostic and prognostic markers in patients with hepatocellular carcinoma. Tumour Biol. 2015;36:7439-7447. doi:10.1007/s13277-015-3430-2

61. Liu M, Liu J, Wang L, et al. Association of serum microRNA expression in hepatocellular carcinomas treated with transarterial chemoembolization and patient survival. PLoS One. 2014;9: e109347. doi:10.1371/journal.pone.0109347

62. Li L, Chen J, Chen X, et al. Serum miRNAs as predictive and preventive biomarker for pre-clinical hepatocellular carcinoma. Cancer Lett. 2016;373:234-240. doi:10.1016/j.canlet.2016.01.028

63. Karakatsanis A, Papaconstantinou I, Gazouli M, Lyberopoulou A, Polymeneas G, Voros D. Expression of microRNAs, miR-21, miR-31, miR-122, miR-145, miR-146a, miR-200c, miR-221, miR-222, and miR-223 in patients with hepatocellular carcinoma or intrahepatic cholangiocarcinoma and its prognostic significance. Mol Carcinog. 2013;52:297-303. doi:10.1002/mc.21864

64. Yao B, Xue Y, Liu Z, Xu M, Tu K, Wang J. [Over-expression of miR-141 inhibits the proliferation, invasion and migration of hepatocellular carcinoma MHCC-97H cells]. Xi Bao yu Fen Zi Mian Yi xue Za Zhi. 2016;32:1083-1087.
65. Meng L, Yang L, Zhao X, et al. Targeted delivery of chemotherapy agents using a liver cancer-specific aptamer. PLoS One. 2012;7: e33434. doi:10.1371/journal.pone.0033434

66. Shi L, Wu L, Chen Z, et al. MiR-141 Activates Nrf2-Dependent Antioxidant Pathway via Down-Regulating the Expression of Keap1 Conferring the Resistance of Hepatocellular Carcinoma Cells to 5-Fluorouracil. Cell Phys Biochem. 2015;35:2333-2348. doi:10.1159/000374036

67. Liang HH, Wei PL, Hung CS, et al. MicroRNA-200a/b influenced the therapeutic effects of curcumin in hepatocellular carcinoma (HCC) cells. Tumour Biol. 2013;34:3209-3218. doi:10.1007/s13277-0130891-z

68. Kulkarni B, Kirave P, Gondaliya P, et al. Exosomal miRNA in chemoresistance, immune evasion, metastasis and progression of cancer. Drug Discov Today. 2019;24:2058-2067. doi:10.1016/j. drudis.2019.06.010

69. Kim MK, Moon YA, Song CK, Baskaran R, Bae S, Yang SG. Tumorsuppressing miR-141 gene complex-loaded tissue-adhesive glue for the locoregional treatment of hepatocellular carcinoma. Theranostics. 2018;8:3891-3901. doi:10.7150/thno.24056

\section{Publish your work in this journal}

OncoTargets and Therapy is an international, peer-reviewed, open access journal focusing on the pathological basis of all cancers, potential targets for therapy and treatment protocols employed to improve the management of cancer patients. The journal also focuses on the impact of management programs and new therapeutic agents and protocols on patient perspectives such as quality of life, adherence and satisfaction. The manuscript management system is completely online and includes a very quick and fair peer-review system, which is all easy to use. Visit http://www.dovepress.com/ testimonials.php to read real quotes from published authors 